\title{
Some Clinical and Laboratory Signs Indicating External Compression of a Nerve Trunk in Leprosy: Details and Rationale
}

\author{
DINKAR D. PALANDE \\ Sacred Heart Hospital, Sakkottai 612401, Kumbakonam \\ Tamil Nadu, South India
}

\begin{abstract}
The details and rationale of clinical signs which when positive indicate the presence of active external compressive and traumatic factors affecting an inflammed nerve trunk in leprosy are described. A new laboratory test suggesting peripheral vascular insufficiency and indicating the need of posterior tibial decompression is also described. The increasing trend towards nerve surgery in leprosy is reviewed and the need for proper selection of cases is stressed.
\end{abstract}

Neuritis and nerve damage in leprosy, in its etiopathology, has two essential components, the effects of the bacillary infection (including immune mechanisms) and the effects of local traumatizing factors on the affected nerve. The latter can be prevented, or their effects ameliorated, by surgery. When a nerve passes through a rigid tunnel this situation predisposes to mechanical compression and trauma, especially subsequent to nerve enlargement. The situation is worsened when nerve inflammation and oedema supervene. As most of these tunnels are on the level of a joint, the joint movements provide further trauma to such a nerve. The sites of nerve involvement in leprosy seem to be restricted to such vulnerable positions. This communication presents the details and rationale of some clinical and laboratory tests which indicate the active presence of such compressive and traumatic factors. These tests when positive are an indication in the opinion of the author, that nerve surgery is likely to be beneficial in these cases, especially when these signs persist in spite of medical treatment including a short course of prednisolone.

\section{THE STRETCH SIGN}

\section{Ulnar Nerve}

The sign is positive when there is pain in the ulnar nerve behind the elbow on flexion of the elbow. Depending on the severity, 3 grades are recognized;

(i) The ulnar nerve pain is elicited only when the elbow is flexed passively just beyond its full active range.

\footnotetext{
*Received for publication 22 May, 1975.
} 
(ii) There is pain in the ulnar nerve on active flexion of the elbow but without any flexion restriction;

(iii) There is flexion restriction of the elbow, usually beyond $100^{\circ}$, because of the pain in the ulnar nerve.

Rationale-normally the increase in length of the extensor surface of the upper limb during elbow flexion is compensated by medial movement of the ulnar nerve in its tunnel behind the elbow. This is similar to the compensatory movement volarwards of the lateral bands of the extensor expansion at the proximal interphalangeal joint level during finger flexion. This essential excursion of the nerve is restricted when the nerve is thickened. Further elbow flexion now results in lengthening of the nerve. As a nerve cannot really be stretched or elongated, this results in trauma to the nerve which is expressed by pain in the nerve on elbow flexion. Hence the term "The Stretch Sign". This sign was first described by Marneffe in 1928 as mentioned by Prof. Diez (1942).

\section{THE TUNNEL COMPRESSION SIGN}

When the patient is asked to contract the flexor carpi ulnaris and flexor superficialis against resistance, (flexion and ulnar deviation of the wrist with the fingers fully flexed), he complains of pain in the ulnar nerve behind the elbow.

Rationale-the roof of the ulnar tunnel behind the elbow is formed by the aponeurosis of origin of the flexor carpi ulnaris from the medial epicondyle and the olecranon. The upper arching fibres of the flexor superficialis also take part in the formation of this roof. The upper one inch or so of the flexor carpi ulnaris muscle through which the ulnar nerve passes below the elbow also can compress the nerve. Contraction of these muscles can cause compression of an already inflamed and oedematous ulnar nerve in leprosy, thereby producing pain.

\section{PAINFUL DISLOCATING NERVE}

When this sign is positive, on flexion of the elbow the ulnar nerve dislocates and in complete flexion lies anteromedial to the epicondyle and the patient complains of pain.

Rationale-in 5\% of normal individuals, a dislocating ulnar nerve can be observed, (WHO report, 1960). However, when the ulnar nerve is enlarged and inflamed, this friction-trauma during dislocation causes additional injury and hence the pain.

\section{Lateral Popliteal Nerve}

\section{STRETCH SIGN}

Restriction of knee extension because of pain in the lateral popliteal nerve in its upper course constitutes the sign. The rationale is similar to that of stretch sign in the ulnar nerve. Usually when the sign is present the lateral popliteal nerve is found to be very markedly thickened and tender.

\section{Posterior Tibial Nerve}

\section{THE RETROMALLEOLAR SIGN}

This is elicited by noticing that the retromalleolar space (behind the medial malleolus) which is normally concave becomes filled up or may even become convex, the swelling being nonfluctuant. 
Rationale-the thickened, inflamed posterior tibial nerve secondarily compresses the posterior tibial vessels and there is associated venous and lymphatic stasis due to tunnel compression. The vascular changes are well documented by Carayon (1971).

\section{LABORATORY TESTS}

A higher total leucocy te count in the toes as compared to that in the peripheral venous blood: the test is considered positive when the total leucocyte count, is considerably higher in the toes than in the venous blood. A difference of at least more than 500 per $\mathrm{mm}^{3}$ is considered significant.

Rationale-we have been doing this test since 1972 af ter coming across the work of Czaczkes and Drefuss (1957) who have shown such increase in the leucocyte count in the peripheral blood in conditions of peripheral vascular insufficiencies. They found this to be true in cases of Reynaud's disease, ergot and phosphorous poisonings, frost bite and similar conditions characterized by peripheral vascular insufficiency. We have found that in cases of posterior tibial neuritis similar findings are obtained. For example in 22 cases which had posterior tibial neuro-vascular decompression; either for posterior tibial neuritis alone or together with an intractable planter ulcer, in our hospital during 1974, the findings were:

1. A higher leucocyte count in the toes was noted in all. The difference between the total leucocyte count in the toes and in the peripheral venous blood was more than 1000 per $\mathrm{mm}^{3}$ in 9 cases, $700-1000$ in 3 cases, 500-700 in 3 cases and less than 500 in 6 cases, no data in one case.

2. In 18 of these the investigation was repeated post-operatively and the findings were: in 8 cases, the difference in the 2 counts was less than 500 ; in another 8 cases, the difference was more than 500. In 4 of these patients even though the toe count was higher than that in the peripheral venous blood, the difference was less than pre-operatively; in 2 cases, the count in the toes was higher than the pre-operative figure. In all these cases with higher than 500 per $\mathrm{mm}^{3}$ leucocyte count in the toes pre-operatively, at operation marked compression of the neurovascular bundle was noted. The operation findings in such cases were described in detail by the author $(1973 ; 1975)$.

A higher count in the toes we take as an indication of distal peripheral vascular insufficiency. Carayon (personal communication) has found a higher red cell count in the toes in similar cases and according to him that indicates a congestive condition in the foot because of autonomic (sympathetic) disturbance caused by compression or damage to the posterior tibial nerve. The insufficiency of blood supply to the foot in these cases is caused by compression of the posterior tibial artery in the retromalleolar tunnel secondary to increase in the contents of the tunnel caused by the enlarged posterior tibial nerve. Often, as seen during the decompression operation, the artery has a tortuous course, and it often bends at the level of the medial malleolus and lies between the bone and the nerve, in which situation, it is more likely to be compressed. As pointed out by Carayon (1964) there is often hypersympathetic activity secondary to neurovascular compression associated not only with peripheral vasoconstriction but also with opening out of shunts which further diminish the distal blood flow. Peripheral venous stasis also probably occurs if the often seen engorgement of the veins accompanying the posterior tibial artery is considered. Local oedema (around ankle) is also frequently seen. This also provides the rationale of periarterial sympathectomy. 


\section{Discussion}

There is an increasing interest in nerve surgery in leprosy as evinced by the number of papers published on the subject from different centres in the last decade. The physiopathological basis corroborated by clinical and contrast radiographic findings (neuro-lymphangio and angio-graphies) has been well propounded by Carayon (1971) and others. At the Xth International Leprosy Congress in Bergan, in the words of S. G. Browne (1973) "A very fruitful discussion took place on the matter of operating on the acutely inflamed peripheral nerve". Among the various published works, Callaway et al. (1964), Vaidyanathan and Vaidyanathan (1968), Parikh et al. (1968), Gabel (1973) and Palande (1973), report varying grades of clinical recovery by surgery (in rather unselected cases) in more than 50\% of nerves. Carayon (1973) while presenting his results of direct nerve trunk surgery on 568 nerves at the Bergan International Conference reported clinical recovery in $79 \%$ of cases operated within 3 months and in $65 \%$ when operated within the first 6 months. Carayon further stressed the need of proper selections of cases. Enna and Jacobson (1974) while reviewing all neurolysis cases done in Carville in 12 years, report: "we believe that overall the surgery was beneficial in terms of lessening the problem of chronic neuritic pain and preventing further paralysis and deformity". Antia (1974) also agrees that "careful and complete release of the thickened nerve may not only relieve the pain, but may also result in recovery of sensory and motor function". He further points out that inadequate appreciation of the existing pathology and of the physical factors responsible for nerve damage is probably the main reason for the conflicting reports and views; to which can be added, an inadequate appreciation of the limitations of surgery. The above stresses the need of proper case selection as also the necessity to curb over-enthusiasm either for, or against, nerve surgery. The significance of the higher leucocyte count in the toes as described is open to question because of the number of factors-physiological, technical and others-influencing the results of this investigation. However, it can definitely be used as an aid to decide if surgical decompression of the posterior neurovascular bundle is required or not. The other clinical signs described definitely imply the presence of an external compressing factor the release of which is very likely to benefit the patient and aid in nerve recovery.

\section{References}

Antia, N. H. (1974). The significance of nerve involvement in leprosy. J. Plas. Reconst. Surg. $54,55$.

Browne, S. G. (1973). Congress highlights and reflections. Lepr. Rev. 44, 161.

Calloway, J. C., Fite, G. L. and Riordan, D. C. (1964). Ulnar and median neuritis due to leprosy, report of 100 cases treated surgically. Int. J. Lepr. 32, 285.

Carayon, A., Bourrel P. and Languillon, J. (1964). Surgery in Leprosy. Paris: Masson et Cie. p. 72 .

Carayon, A. (1971). Physiopathologie de la Neurite Hansenienne Et bases. Ther. med. Tropicale. 31, 503.

Carayon, A. and Giraudeau, P. Case selection and results of direct surgery in 586 cases of leprous neuritis involving the large nerve trunks. Paper presented at the 10 th International Leprosy Congress, 1973; Abstracts of the above Conference, p. 172.

Czaczkes, J. W. and Dreyfuss, F. (1957). Discrepancy of finger tip and earlobe leucocyte count in Raynaud's disease. Am. J. Med.Sci. 234, 325.

Diez, L. (1942). Ann. Cirurgia, (Portuguese). (vide personal communication, Carayon). 
Enna and Jacobson. (1974). Neurolysis for leprous neuritis. Int. J. Lepr. 42, 162.

Galal, Z. Said, Zohdy, A. and EL-Akkan, I. N. (1973). External and Internal neurolysis of ulnar and median nerves in leprous neuritis. Lepr. Rev. 44, 36.

Palande, D. D. (1973). A review of twenty three operations on the Ulnar nerve in leprous neuritis. J. Bone Jr. Surg. 55-A, 1457.

Palande, D. D. and Azhaguraj, M. (1975). Surgical decompression of posterior tibial neurovascular complex in treatment of certain chronic plantar ulcers and posterior tibial neuritis in leprosy. Paper presented at 25th All India Leprosy Workers Conference at Wardha, India, in October 1973. Int. J. Lepr. 43, 36.

Parikh, A. C., Ganapathy, R. and Kothare, K. B. (1968). Decompression of ulnar and median nerves in leprous neuritis. Lep. Rev. 39, 143.

Vaidyanathan, E. P. and Vaidyanathan, S. I. (1968). Treatment of early ulnar neuritis and early ulnar palsy, Lepr. Rev. 39, 217.

W.H.O. Report 221. (1960). Scientific Meeting on Rehabilitation in Leprosy; p. 12. Vellors, India. 